\title{
POSITRON ANNIHILATION IN AND COMPRESSIBILITY OF WATER-ORGANIC MIXTURES. THE SYSTEM WATER-FORMAMIDE
}

\author{
K. Jerie, A. Baranowski \\ Institute of Experimental Physics, University of Wrocław \\ Pl. Maxa Borna 9, 50-205 Wrocław, Poland \\ J. GlińsKi AND K. ORZEChowski \\ Institute of Chemistry, University of Wrocław, Poland \\ (Received July 22, 1993; revised version October 13, 1993)

\begin{abstract}
The experimental results of positron annihilation experiments in aqueous solutions of formamide were compared to those of ultrasonic velocity. The systems under investigation behave untypically while changing formamide concentration. The results are explained in terms of hydrophilic hydration and lack of hydrophobic interactions.
\end{abstract}

PACS numbers: $61.25 . \mathrm{Em}, 78.70 . \mathrm{Bj}$

\section{Introduction}

In the papers of this series we investigated the structure of aqueous solutions of non-electrolytes: tetrahydrofuran [1], cyclohexylamine [2], methyl alcohol [3], ethyl alcohol [4], n-propyl alcohol [5], isopropyl alcohol [6], dimethylsulfoxide [7], and pyridine [8]. So far, however, all the solutes investigated have had hydrophobic parts, and their interactions with water framework could be explained in terms of hydrophobic hydration. $[9,10]$. Encouraged by some success in comparing of two independent experimental results, positron annihilation and ultrasonic velocity, we now try to test the title system, where no hydrophobic hydration should occur and any interactions should have hydrophilic character only.

It is obvious from the results of our previous positron annihilation experiments that all the systems investigated exhibit characteristic dependencies of annihilation parameters on concentration of nonelectrolyte in water. In all the systems their spectra could be resolved into two compounds: long- and short-living ones. They can be interpreted assuming two possible mechanisms of positronium annihilation in liquid. The long-living component of the spectrum corresponds to ortho-positronium annihilation in the pick-off process, while the short-living one 
- to free positron annihilation as well as that of para-positronium. The two processes will be denoted using subscript 1 for the short, and 2 for the long lifetimes. All the details of treatment of the annihilation data are given in details in other papers of this series $[1,2,4,7]$. The resolution is proceeded with respect to the condition $I_{1}+I_{2}=100 \%$, where $I_{1}$ and $I_{2}$ are the intensities of the short- and long-living components.

After the resolution, both the components are described by two parameters: lifetimes $\tau_{1}$ and $\tau_{2}$, and intensities $I_{1}$ and $I_{2}$. Usually only the long-lived parameters, i.e. $\tau_{2}$ and $I_{2}$, are used for interpretation of liquid structure. Formation, as well as lifetime of ortho-positronium, is strongly influenced by the physical properties of the medium surrounding the positronium atom. The liquid water structure, with cavities and open hydrogen-bonded network, can be modified and then stabilized by presence of guest molecule. The nonelectrolytes form strong hydrates of quasi-clathrate architecture, where considerably big empties are occupied by solute molecules. Thus, the liquid structure becomes more open-work, but also more rigid. The competition of these two effects yields characteristic concentration dependence of $t_{2}$ and $I_{2}$ : the former exhibits an inflection and the latter - a maximum of the concentration corresponding to the stoichiometry of the hydrates formed.

One can read the same stoichiometry from ultrasound velocity measurements. From Laplace's equation $\beta=\left(\rho c^{2}\right)^{-1}$ the adiabatic compressibility coefficient $\beta$ can be calculated ( $\rho-$ density of liquid, $c$ - sound velocity). When plotting the adiabatic compressibilities $\beta$ against nonelectrolyte concentration in water, one sometimes observes that isotherms of $\beta$ intersect at a common point. The $X$-coordinate of this point gives stoichiometry of the quasiclathrate hydrates, according to the concept of Endo [11]. The details of this interpretation are given in Refs. [12] and [13]. It is worth noting that the compressibility value at the crossing point is, in most cases, very close to that found by von Stackelberg for the solid clathrates [i4].

The series of our previous papers concerned the systems of aqueous solutions of nonelectrolytes, all of them containing hydrophobic part of molecule, what allows the hydrophobic hydration. The two different experimental methods used, i.e. positron annihilation and sound velocity, yielded similar results, explainable in terms of structure-breaking concept. The stoichiometries of hypothetical quasiclathrate hydrates were similar, too, and independent on the method.

In this paper we investigated the system water + formamide $\left(\mathrm{HCONH}_{2}\right)$. The solute is different from those used before: there is no distinct hydrophobic part in the molecule of formamide and, consequently, only hydrophilic hydration should be possible. It seemed interesting how differs the experimental picture of a system without hydrophobic hydration from those investigated before.

\section{Experimental}

\subsection{Chemicals}

Formamide (analytical grade) from Romil, Great Britain, was used without further purification. Freshly prepared, double distilled water was used. Solutions were prepared by weighing. 


\subsection{Positron annihilation measurements}

The annihilation measurements were carried out at room temperature (approx. $25^{\circ} \mathrm{C}$ ) with a standard measuring device based on the "fast-slow" coincidence technique as described elsewhere [1].

\subsection{Sound velocity measurements}

The ultrasonic velocity was measured with an accuracy of $0.1 \%$ using a "sing-around" equipment. The details of the apparatus and measurement techniques applied in this study are given in Ref. [15].

\subsection{Density measurements}

The density was measured using a calibrated sinker method. Calibrations were determined daily using the known densities of air and water. The reproducibilities were $0.01 \mathrm{~kg} / \mathrm{m}^{3}$.

\section{Experimental results}

\subsection{Sound velocity}

The adiabatic compressibility coefficients $\beta$ were calculated from Laplace's equation

$$
\beta=\left(\rho c^{2}\right)^{-1},
$$

where $\rho$ - density of the liquid, $c-$ sound velocity. The concentration dependence of the compressibility in the low nonelectrolyte concentration region, which is of interest for further discussion, is shown in Fig. 1.

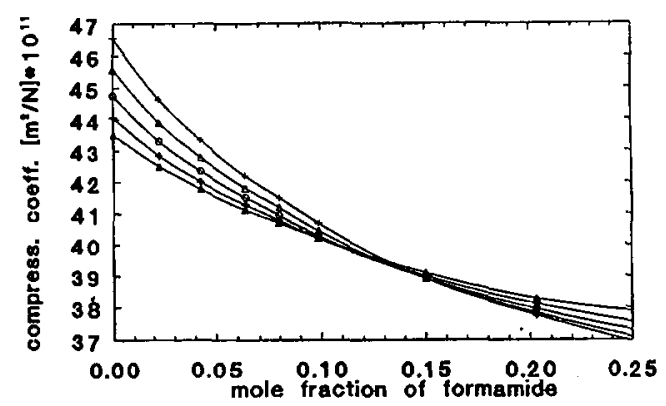

Fig. 1. Adiabatic compressibility coefficients vs. formamide concentration for different temperatures: $+288 \mathrm{~K}, \Delta 293 \mathrm{~K}, \circ 298 \mathrm{~K},+303 \mathrm{~K}, \Delta 308 \mathrm{~K}$.

The adiabatic compressibility plotted against the mole fraction of formamide (Fig. 1) shows that there is no common intersection point of isotherms of adiabatic compressibility. The isotherms intersect depending on temperature at mole fraction of the solute varying from 1.1 to 1.3 (in the temperature range studied). This result is different comparing with the known aqueous solutions of nonelectrolytes with hydrophobic group [1-8]. 


\subsection{Positron mean lifetimes}

The numerical treatment briefly described above yielded the positron annihilation parameters $\tau_{1}, \tau_{2}$ and $I_{2}$. Concentration dependencies of $\tau_{2}$ and $I_{2}$ are collected in Figs. 2 and 3.

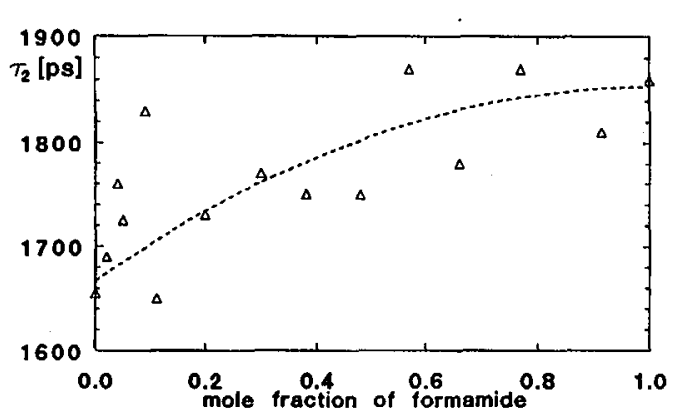

Fig. 2. The dependence of $\tau_{2}$ (the lifetime of the long-living component of annihilation spectrum) on formamide concentration in water. The line is arbitrary (second order polynomial).

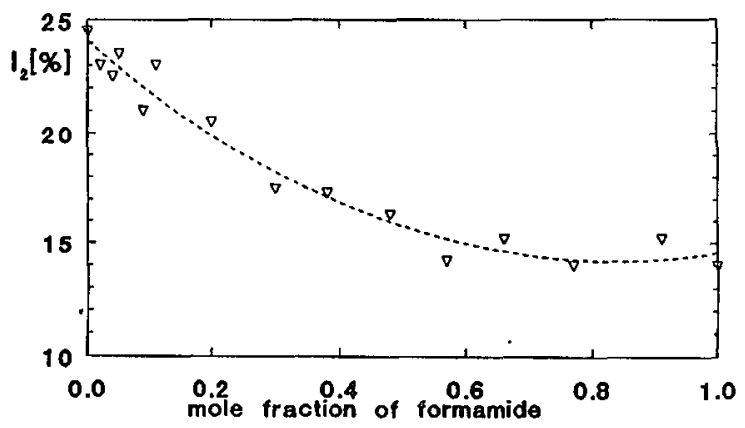

Fig. 3. The dependence of $I_{2}$ (the intensity of the long-living component of annihilation spectrum) on formamide concentration in water. The line is arbitrary (second order polynomial).

Considerably big relative error of $\tau_{2}$ should be noted, resulting from a rather small overall change in this parameter. However, in the limits of the errors, the concentration dependence in Fig. 2 seems to be smooth. The annihilation results differ significantly from those obtained in the aqueous mixtures of normal alcohols and other solutes with hydrophobic group. There is neither inflection of $\tau_{2}$ nor maximum of $I_{2}$, which could be observed in the hydrophobically hydrated systems at concentrations corresponding to stoichiometries of liquid quasicrystalline hydrates. 


\section{Discussion}

The formation of clathrate hydrates in the aqueous solutions of nonelectrolytes with hydrophobic parts of their molecule is well proved now. According to the concept of iceberg formation of Frank and Evans [9] or to that of hydrophobic hydration formulated by Franks [10], when an organic compound is dissolved in water, the water structure is modified around the nonpolar parts of the solute molecule in the direction of greater "crystallinity", whatever the actual quasi-solid water structure induced in this way. In the vicinity of a guest molecule the water structure is determined mainly by its moiety. Molecules of solute are accommodated in the cavities of the open hydrogen-bonded network, which in pure water are most probably occupied by "monomeric" water molecules [16-19].

The system under investigation is different. The lack of hydrophobic hydration means that formamide molecules are rather accommodated in the hydrogen-bonded structure of water than in its cavities. Such accommodation is possible in every concentration, thus one does not observe any distinguished region of changing structure, while the changes are continuous.

The systems, where solute molecules have no hydrophobic group, can be structure-breakers, similar to those with the hydrophobic part. Negative deviations in $\varepsilon$ vs. composition curves ( $\varepsilon$ - relative permittivity) reported by Corradini et al. [20] for the system water + ethane-1, 2-diol is an example of the system where no hydrophobic hydration occurs. The authors interpret their results in terms of formation of strong hydrogen bondings between diol and water molecules, as well as between molecules of the components.

A good example of interpretational problems in the cases of water-nonelectrolyte systems (where the solute has no hydrophobic part) is the system water-urea. Recently, Boek and Briels [21] investigated it theoretically using molecular dynamics simulations and comparing the results with those from neutron scattering experiments performed by Finney and Turner [22]. In contrary to the previous models of Frank and Franks [23], Schellman [24] (refined by Kreschek and Scheraga [25]) and Stokes [26], Boek and Briels conclude that for water-urea system of considerably high urea concentration ( 2 molal), the presence of dimers is very improbable. Of course, this conclusion should be valid for lower concentrations, too.

Applying this result to the title system it means that the hydration of formamide in aqiveous solutions is not similar to the solutions where strong hydrophobic hydration occurs. The structural changes are smooth, since the solute molecules are accommodated in water network. The concentration of formamide corresponding to (not exact) interception of compressibility isotherms and equal to (approximately) $0.11-0.13$ mole fraction of formamide is considerably higher than that corresponding to formation of type I clathrates hydrates (like in the system water-methanol [3]). It is interesting, however, that the value of compressibility at this concentration $\left(\beta \simeq 40 \times 10^{11} \mathrm{~m}^{2} / \mathrm{N}\right)$ is almost the same as for solid clathrate lyydrates measured by von Stackelberg [14]. It seems probable that at mole fraction of formamide roughly equal to 0.12 , the solution contains mainly a mixture of hydrated solute molecules, and the average hydration number is equal to 6-8. 
The concentration dependence of positron annihilation parameters in Figs. 2 and 3 confirms the above considerations. The lack of drastical structure changes results in the observed smooth dependencies, with no inflections and extrema.

\section{Conclusions}

The formamide molecule contains no hydrophobic part, implying gradual structural changes in solution while adding this solute to water. No clathrate-like hydrates are formed and the concentration dependencies of positron annihilation parameters are smooth. The formation of dimers of the solute is also rather improbable.

\section{Acknowledgment}

The financial support no. PB 1168/2 from the Committee for Scientific Research is acknowledged.

\section{References}

[1] K. Jerie, A. Baranowski, B. Rozenfeld, S. Ernst, J. Gliński, Acta Phys. Pol. A 64, 77 (1983).

[2] K. Jerie, A. Baranowski, B. Rozenfeld, B. Jeżowska-Trzebiatowska, S. Ernst, J. Gliniski, Acta Phys. Pol. A 66, 3 (1984).

[3] K. Jerie, A. Baranowski, B. Rozenfeld, S. Ernst, J. Glinski, Acta Phys. Pol. A 66, 167 (1984).

[4] K. Jerie, A. Baranowski, S. Ernst, J. Gliński, Acta Phys. Pol. A 69, 81 (1986).

[5] K. Jerie, A. Baranowski, S. Ernst, J. Glinski, Acta Phys. Pol. A 69, 91 (1986).

[6] K. Jerie, A. Baranowski, B. Rozenfeld, J. Glinski, S. Ernst, Acta Phys. Pol. A 73, 667 (1988).

[7] K. Jerie, A. Baranowski, B. Rozenfeld, B. Jeżowska-Trzebiatowska, J. Gliński, Acta Phys. Pol. A 79, 507 (1991).

[8] K. Jerie, A. Baranowski, B. Rozenfeld, B. Jeżowska-Trzebiatowska, J. Glinski, Acta Phys. Pol. A 82, 183 (1992).

[9] H.S. Frank, M.W. Evans, J. Phys. Chem. 13, 507 (1945).

[10] F. Franks, Ann. N. Y. Acad. Sci. 125, 277 (1965).

[11] H. Endo, Bull. Chem. Soc. Jpn. 16, 1586 (1973).

[12] S. Ernst, J. Gliński, Polish J. Chem. 56, 339 (1982).

[13] S. Ernst, J. Gliński, Mater. Sci. 3, 68 (1977).

[14] M. von Stackelberg, H. Müller, Z. Elektrochem. 58, 25 (1954).

[15] S. Ernst, J. Glinski, B. Jeżowska-Trzebiatowska, Acta Phys. Pol. A 64, 501 (1979).

[16] L. Pauling, in: Hydrogen Bonding, Eds. D. Hadzi, H.W. Thompson, Pergamon, London 1959, p. 127.

[17] H.S. Frank, A.S. Quist, J. Chem. Phys. 34, 604 (1961).

[18] V.N. Yashkitshev, O.Ya. Samoilov, Zh. Strukt. Khim. 3, 211 (1962). 
[19] M.N. Buslayeva, O.Ya. Samoilov, Zh. Strukt. Khim. 4, 502 (1963).

[20] F. Corradini, L. Marcheselli, L. Tassi, G. Tosi, J. Chem. Soc. Faraday Trans. 89, 123 (1993).

[21] E.S. Boek, W.J. Briels, J. Chem. Phys. 98, 1422 (1993).

[22] J.L. Finney, J. Turner, Electrochim. Acta 9, 1183 (1988).

[23] F.S. Frank, F. Franks, J. Chem. Phys. 48, 4746 (1968).

[24] J.A. Schellman, C. R. Trav. Lab. Carlsberg, Ser. Chim. 29, 223 (1955).

[25] G.C. Kreschek, H.A. Scheraga, J. Phys. Chem. 69, 1704 (1965).

[26] R.H. Stokes, Aust. J. Chem. 20, 2087 (1967). 